\title{
Registros inéditos de borboletas (Papilionoidea e Hesperioidea) ameaçadas de extinção para o Estado do Paraná, Brasil: novos subsídios para reavaliação dos critérios de ameaça
}

\author{
Diego Rodrigo Dolibaina ${ }^{1,2}$, Eduardo Carneiro ${ }^{1}$, Fernando Maia Silva Dias ${ }^{1}$, \\ Olaf Hermann Hendrik Mielke ${ }^{1}$ \& Mirna Martins Casagrande ${ }^{1}$ \\ ${ }^{1}$ Laboratório de Estudo de Lepidoptera Neotropical, Departamento de Zoologia, \\ Universidade Federal do Paraná - UFPR, CP 19020, CEP 81531-980, Curitiba, PR, Brasil \\ ${ }^{2}$ Autor para correspondência: Diego Rodrigo Dolibaina, e-mail: dirodrido@hotmail.com
}

\begin{abstract}
DOLIBAINA, D.R., CARNEIRO, E., DIAS, F.M.S., MIELKE, O.H.H. \& CASAGRANDE, M.M. Unpublished records of threatened butterflies (Papilionoidea and Hesperioidea) to Paraná State, Brazil: new contributions for the evaluation of threat criteria. Biota Neotrop. 10(3): http://www.biotaneotropica.org.br/v10n3/en/abstr act?article+bn01210032010.
\end{abstract}

\begin{abstract}
Many countries regard the red lists of threatened species as useful tools for promoting the biological conservation. However, little effort has been made to gather base-line information on the presence and distribution of such species, or their conservation. Therefore, the present work provides new records of some threatened butterflies species distributed through Paraná, one of which is already included in the Brazilian red list, and thus complementing the knowledge of geographical distribution and conservation. In total, 15 new records belonging to 8 species are given, confirming past records, or representing new distribution records. The species addressed in our study are: Passova passova practa Evans, 1951 (CR), Zonia zonia diabo Mielke \& Casagrande, 1998 (VU Brazil), Alesa prema (Godart, [1824]) (VU), Symmachia arion (C. Felder \& R. Felder, 1865) (VU), Cyanophrys bertha (Jones, 1912) (EN), Euryades corethrus (Boisduval, 1836) (EN), Charonias theano (Boisduval, 1836) (EN), and Pampasatyrus glaucope glaucope (C. Felder \& R. Felder, 1865) (VU). Except for Charonias theano and Pampasatyrus glaucope glaucope, all species were recorded on the past three years (2007-2009). Approximately one-half of the records included here correspond to small conservation units located at the northwest and northeast regions of Paraná, while records from central-south region correspond to non-protected areas, specially the endemic grasslands species. From these new data, we suggest the following modifications to threat status: remove P. p. practa, C. bertha and S. arion from the red list. Z. z. diabo should be considered critically endangered (CR), while the status of $E$. corethrus should be revised to critically endangered (CR). We make no recommendations for change in status for A. prema, P. g. glaucope and C. theano.

Keywords: conservation, Lepidoptera, red list, distribution, conservation units.
\end{abstract}

DOLIBAINA, D.R., CARNEIRO, E., DIAS, F.M.S., MIELKE, O.H.H. \& CASAGRANDE, M.M. Registros inéditos de borboletas (Papilionoidea e Hesperioidea) ameaçadas de extinção para o Estado do Paraná, Brasil: novos subsídios para avaliação dos critérios de ameaça. Biota Neotrop. 10(3): http://www. biotaneotropica.org.br/v10n3/pt/abstract?article+bn01210032010.

Resumo: Hoje a presença de listas vermelhas de espécies ameaçadas de extinção é comum em muitos países que as julgam necessárias para promover a conservação da natureza. No entanto, poucos esforços vêm sendo realizados com objetivo de resgatar as informações básicas acerca da presença e distribuição dessas espécies, sobretudo sua conservação. Portanto, o presente trabalho fornece novos registros de borboletas consideradas ameaçadas de extinção com distribuição no Paraná, incluindo uma da lista vermelha brasileira e complementando assim o conhecimento de suas distribuições geográficas e estudos de conservação. Ao total, somam-se 15 registros inéditos correspondentes a oito espécies, confirmando a presença onde já havia registros ou adicionando novos registros de distribuição. As espécies abordadas em nosso estudo são: Passova passova practa Evans, 1951 (CR), Zonia zonia diabo Mielke \& Casagrande, 1998 (VU - Brasil), Euryades corethrus (Boisduval, 1836) (EN), Cyanophrys bertha (Jones, 1912) (EN), Alesa prema (Godart, [1824]) (VU), Symmachia arion (C. Felder \& R. Felder, 1865) (VU), Pampasatyrus glaucope glaucope (C. Felder \& R. Felder, 1865) (VU) e Charonias theano (Boisduval, 1836) (EN). Exceto P. g. glaucope e C. theano, todas foram registradas nos últimos três anos. Metade dos registros corresponde a pequenas unidades de conservação do noroeste e nordeste paranaense, enquanto os fornecidos para região centro-sul não estão protegidos, especialmente as espécies endêmicas dos Campos Naturais. A partir dos novos dados, são sugeridas as seguintes alterações na situação de ameaça das espécies: descaracterização dos graus de ameaça de P. p. practa, C. bertha e S. arion. Z. z. diabo deve ser inserida como criticamente em perigo (CR), assim como E. corethrus deveria elevar sua categoria em perigo (EN) para criticamente em perigo (CR). A. prema, $P$. g. glaucope e $C$. theano não tiveram as categorias e critérios alterados.

Palavras-chave: conservação, Lepidoptera, lista vermelha, distribuição, unidades de conservação. 


\section{Introdução}

A proposição de listas vermelhas de espécies ameaçadas de extinção, em níveis nacional ou estadual, são recorrentes em muitos países que as julgam necessárias para promover ações para a conservação da natureza. A proteção regional de populações de espécies ameaçadas promove a conservação da espécie como um todo, visto que as distinções genéticas entre populações em diferentes locais são preservadas, além de proteger o patrimônio natural de cada estado (Machado et al. 1998). Após o lançamento da primeira lista de fauna ameaçada do Brasil que inclui invertebrados (Bernardes et al. 1990), o Paraná foi o pioneiro a organizar uma lista estadual de Fauna Ameaçada de Extinção, a partir de um grupo de trabalho designado pelo Instituto Ambiental do Paraná em 1991 e cujos resultados foram publicados quatro anos mais tarde (Paraná 1995). Casagrande \& Mielke (1993) publicaram um complemento detalhado sobre os resultados deste grupo de trabalho, do qual participaram, apresentando comentários e ilustrações de todas as espécies. Posteriormente, outros estados acabariam por produzir suas listas, somando atualmente sete estados com listas próprias (Espírito Santo, Minas Gerais, Pará, Rio de Janeiro, Rio Grande do Sul e São Paulo), dentre as quais somente o Rio Grande do Sul não inclui espécies de borboletas (Fontana et al. 2003).

A primeira lista do Estado do Paraná (Paraná 1995) contém 17 espécies de borboletas apresentando alguma situação de ameaça, normalmente justificada pela ausência de registros em coletas realizadas: uma provavelmente extinta, dez consideradas raras e seis de ocorrência provável, pois há registros nos estados vizinhos possuidores de fitofisionomias também representadas no Paraná (Casagrande \& Mielke 1993). Na segunda versão da lista (Mikich \& Bernils 2004), uma revisão da primeira, são relacionadas 15 espécies de Lepidoptera sofrendo algum tipo de ameaça, desconsiderando todas as espécies com ocorrência provável, e acrescentadas quatro outras espécies de borboletas e uma de mariposa (Mielke \& Casagrande 2004).

No entanto, mesmo após a publicação da primeira lista de espécies ameaçadas, pouco de efetivo tem sido feito para resgatar as informações básicas acerca da presença, distribuição e conservação destas espécies. Mielke \& Casagrande (2004) abordam que a primeira medida de conservação dessas espécies seria o encontro de populações remanescentes no estado. Com essa justificativa, este estudo objetiva trazer novos registros para o conhecimento de populações de borboletas consideradas ameaçadas de extinção no estado do Paraná, e consequentemente sugerir novas avaliações a partir das informações coligidas.

\section{Material e Métodos}

Novos registros foram feitos com base em coletas aleatórias nos últimos três anos em diversas localidades do Paraná ou através de exemplares depositados em coleções entomológicas até então não considerados. As áreas de coleta foram visitadas mais de uma vez. Os exemplares coletados recentemente se encontram depositados na Coleção Entomológica Pe. Jesus Santiago Moure (UFPR). A classificação e nomenclatura seguem Lamas (2004). Na sinopse das espécies consideramos: "distribuição geral", a ocorrência conhecida para a espécie excetuando o estado do Paraná; "distribuição conhecida no Paraná" corresponde a dados publicados na última versão da lista vermelha da fauna ameaçada do Paraná (Mielke \& Casagrande 2004) e por fim, "registros inéditos no Paraná" para dados não inclusos na lista vermelha do Paraná. Todos os registros fornecidos são acompanhados de acrônimo do museu ou coleção de depósito, quando não constarem dados publicados, ou de bibliografia. São utilizados os seguintes acrônimos: BMNH - The Natural History Museum, ex
British Museum (Natural History), Londres, Inglaterra; USNM National Museum of Natural History, Washington, USA; DZUP Departamento de Zoologia, Universidade Federal do Paraná, Coleção Entomológica Pe. Jesus Santiago Moure, Curitiba, Paraná, Brasil e CGCM - coleção particular de Carlos G. C. Mielke. As categorias de ameaça utilizadas seguem às propostas pela IUCN (2001). Na Figura 1 estão inclusas todas as unidades de conservação do Paraná, como Parques Nacionais e Estaduais, Estações Ecológicas, Florestas Nacionais e Estaduais, Reservas Biológicas, Reservas Particulares do Patrimônio Natural (RPPN), Refúgios da Vida Silvestre, Parque Florestal, Hortos Florestais e Área Especial de Interesse Turístico (AEIT).

\section{Resultados}

A partir do presente estudo foram ampliados os registros para oito espécies, sendo elas: Passova passova practa Evans, 1951, Zonia zonia diabo Mielke \& Casagrande, 1998, Euryades corethrus (Boisduval, 1836), Cyanophrys bertha (Jones, 1912), Alesa prema (Godart, [1824]), Symmachia arion (C. Felder \& R. Felder, 1865), Pampasatyrus glaucope glaucope (C. Felder \& R. Felder, 1865) e Charonias theano (Boisduval, 1836) (Figura 1).

Abaixo segue uma sinopse da situação de cada espécie, contendo: posição sistemática, categorias e critérios de ameaça, distribuição geral, distribuição conhecida no Paraná, registros inéditos no Paraná, além de informações relevantes acerca de sua biologia. Para as novas ocorrências no Paraná, são fornecidos dados como altitude e data(s) do(s) registro(s).

Passova passova practa Evans, 1951 (Hesperiidae: Pyrrhopyginae) (Figuras 2-5).

Situação atual: CR B2ab(ii,iii)

Distribuição geral: BRASIL: Rio Grande do Sul: Derrubadas (Parque Estadual do Turvo) (DZUP); São Paulo: Campinas (DZUP) e Pereira Barreto (DZUP); Goiás: Leopoldo Bulhões (BMNH); Mato Grosso: Barra do Garças (DZUP) e São Vicente

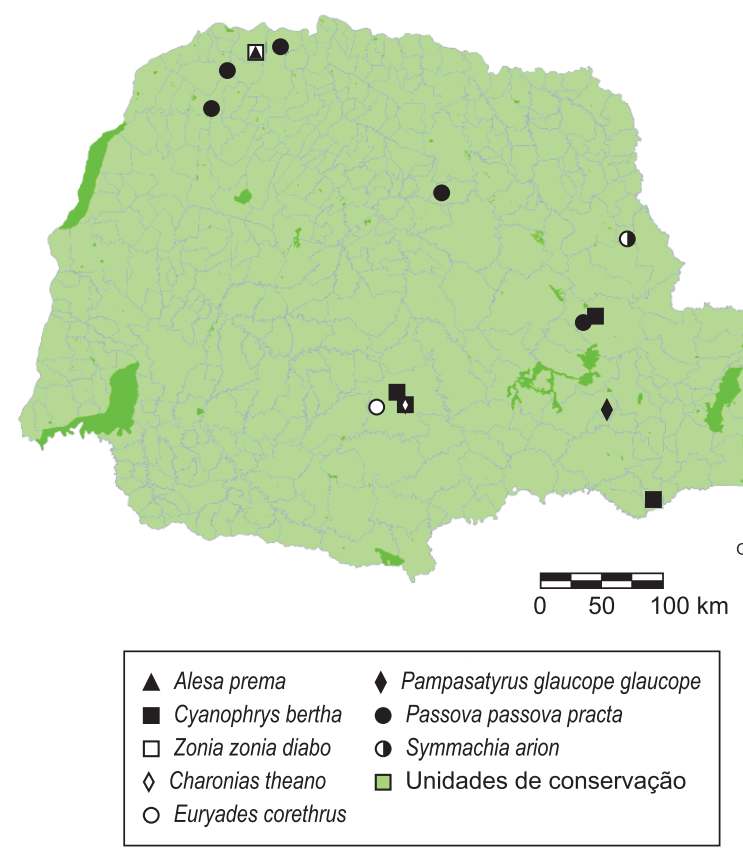

Figura 1. Novos registros de distribuição de borboletas ameaçadas de extinção para o Paraná.

Figure 1. New distribution records of threatened butterflies to Paraná. 
(USNM). ARGENTINA: Misiones: Puerto Iguazu (Núñez Bustos 2009). PARAGUAI: Cordillera: San Bernardino (USNM); Paraguari: Sapucay (BMNH).

Distribuição conhecida no Paraná: Foz do Iguaçu.

Registros inéditos no Paraná: Planaltina do Paraná, RPPN Duas

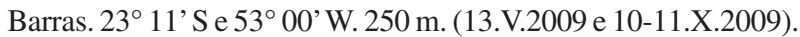
Loanda, RPPN Fazenda Matão. $22^{\circ} 54^{\prime}$ S e $52^{\circ} 52^{\prime}$ 'W. 450-500 m. (22.I.2010). Paranavaí, 12 km a Oeste de Santo Antônio do Caiuá. $22^{\circ} 45^{\prime} \mathrm{S}$ e $52^{\circ} 27^{\prime}$ W. 438 m (2.I.2010). Tamarana (Recanto Pinhão), $23^{\circ} 48^{\prime}$ S e $51^{\circ} 09^{\prime}$ W. 860 m (9.IV.2006). Castro (sem localidade exata) (BMNH - holótipo), $24^{\circ} 47^{\prime} \mathrm{S}$ e $50^{\circ} 00^{\prime} \mathrm{W}$. $984 \mathrm{~m}$.

Biologia: adultos, principalmente fêmeas, foram observados voando pelas trilhas e bordas do fragmento de Floresta Estacional Semidecidual, seu habitat, na RPPN Duas Barras, entre as 11:00 e 15:00 horas, ou esporadicamente pousados sob a face abaxial de folhas largas da planta hospedeira: Guarea sp. (Meliaceae). Larvas foram encontradas nas outras novas localidades, exceto Castro, sendo criadas para a obtenção e identificação dos adultos. Embora não tenha sido registrada por Fritz Plaumann, que durante mais de 40 anos viveu em Seara (Nova Teutônia), provavelmente sua distribuição original inclua localidades no oeste do Estado de Santa Catarina.

Sugestão de alteração no critério de ameaça: Com registro em seis localidades (cinco novas) passa a ter uma área de ocupação acima de $2.000 \mathrm{~km}^{2}$, consequentemente descaracterizando-a como uma espécie ameaçada. A abundância de larvas encontradas em sua planta hospedeira sugere que a suposta raridade seja um artifício de coleta.

Zonia zonia diabo Mielke \& Casagrande, 1998 (Hesperiidae: Pyrrhopyginae) (Figuras 6-7).

Situação atual: VU (lista nacional) B2ab(ii,iii)

Distribuição geral: BRASIL: São Paulo: Teodoro Sampaio (Morro do Diabo) (Mielke \& Casagrande, 1998); Goiás: Pirenópolis (Mielke \& Casagrande, 1998).

Distribuição conhecida no Paraná: desconhecida.

Registro inédito no Paraná: Terra Rica, Parque Municipal Três Morrinhos. 22 ${ }^{\circ}$ 46' S e 52 39' W. 400-615 m (9.X.2009).

Biologia: Machos foram observados exibindo comportamento de "hilltopping" (Shields 1967, Pe'er et al. 2004), que se caracteriza pelo direcionamento de indivíduos aos topos de
(2)

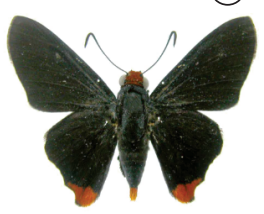

(3)

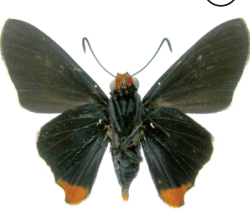

(4)

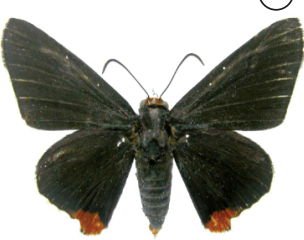

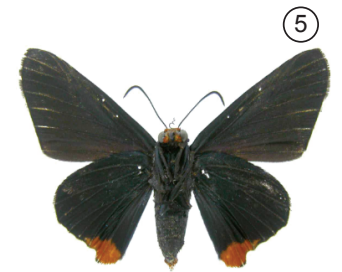

(8)
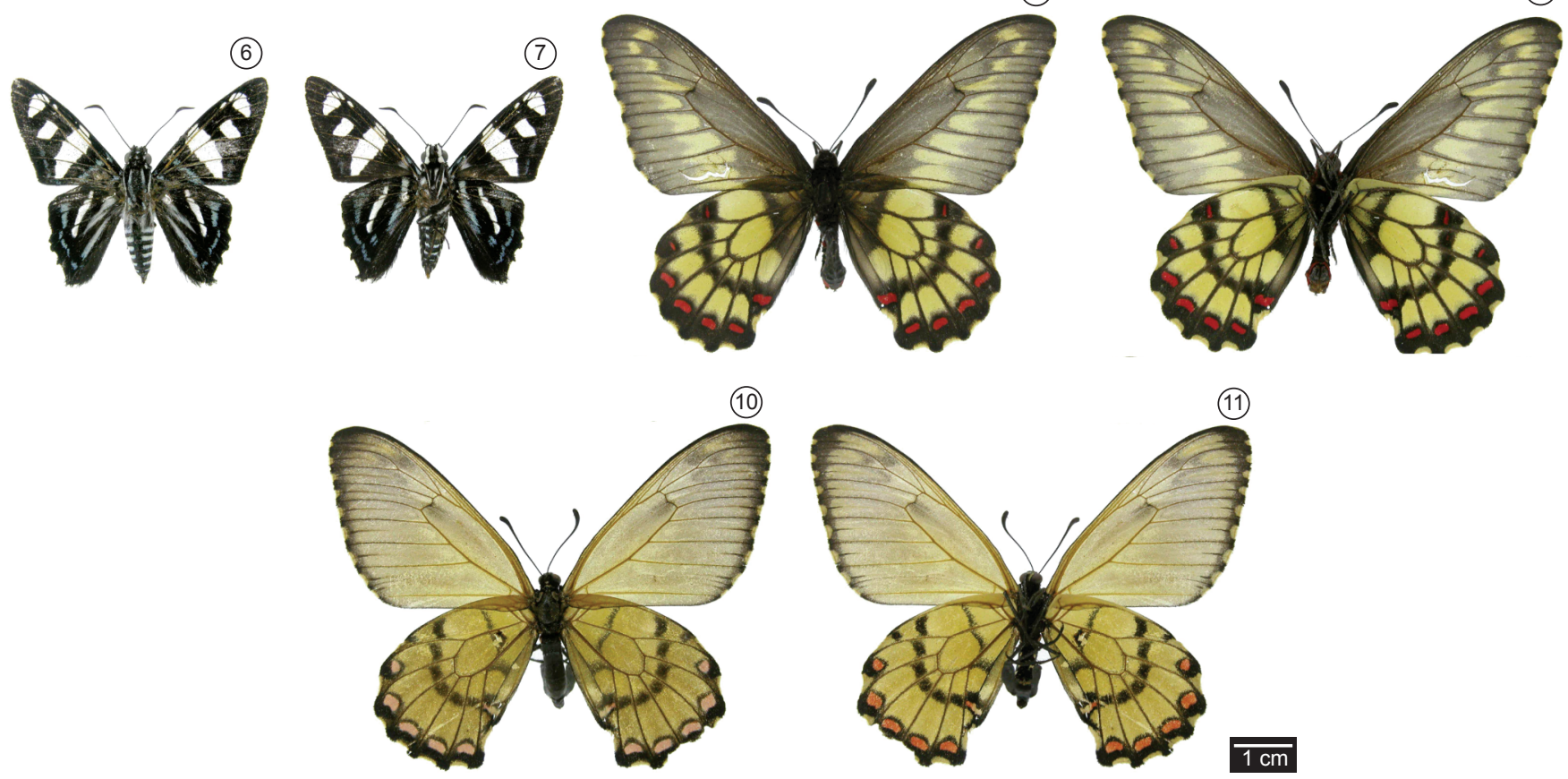

Figuras 2-11. (2-3) Passova passova practa, macho (dorsal e ventral) (RPPN Fazenda Matão, Loanda, ex. larva); (4-5) Passova passova practa, fêmea (dorsal e ventral) (Paranavaí, $12 \mathrm{~km}$ a Oeste de Santo Antônio do Caiuá, ex. larva); (6-7) Zonia zonia diabo, macho (dorsal e ventral) (Parque Municipal dos Três Morrinhos, Terra Rica); (8-9) Euryades corethrus, macho (dorsal e ventral) (Fazenda Três Capões, Guarapuava); (10-11) Euryades corethrus, fêmea (dorsal e ventral) (Fazenda Três Capões, Guarapuava).

Figures 2-11. (2-3) Passova passova practa, male (dorsal and ventral) (RPPN Fazenda Matão, Loanda, ex. larva); (4-5) Passova passova practa, female (dorsal and ventral) (Paranavaí, 12 km a Oeste de Santo Antônio do Caiuá, ex. larva); (6-7) Zonia zonia diabo, male (dorsal and ventral) (Parque Municipal dos Três Morrinhos, Terra Rica); (8-9) Euryades corethrus, male (dorsal and ventral) (Fazenda Três Capões, Guarapuava); (10-11) Euryades corethrus, female (dorsal and ventral) (Fazenda Três Capões, Guarapuava). 
morros para acasalamento. O horário do vôo nesse caso foi observado entre 12:00 e 14:00 horas, já que Parelbella ahira extrema (Röber, 1925), voa a partir das 14:00 horas, também defendendo território no mesmo ponto. Curiosamente são regiões com morros, onde a coleta desta subespécie parece ser facilitada pelo comportamento de hilltopping. Atualmente a subespécie não se encontra na lista estadual devido à ausência de registros no Paraná, embora o município de Teodoro Sampaio seja limítrofe ao estado, aproximadamente $45 \mathrm{~km}$ ao Norte do Parque Municipal de Três Morrinhos. A fêmea ainda é desconhecida, assim como sua planta hospedeira.

Sugestão de alteração no critério de ameaça: Deve ser inserida na lista do Paraná como criticamente em perigo $(\mathrm{CR})$ por ser conhecida de uma única localidade inferior a $10 \mathrm{~km}^{2}$. No entanto, seriam necessários mais estudos de campo, pois a espécie é comum em topos de morros, onde a sua captura é facilitada. Nunca foi registrada em áreas planas, provavelmente por habitar o dossel, onde não é possível amostrá-la.

Euryades corethrus (Boisduval, 1836) (Papilionidae: Papilioninae) (Figuras 8-11).

Situação atual: EN B2ab(ii,iii)

Distribuição geral: BRASIL: Rio Grande do Sul: Gravataí (Biezanko, 1959), Itaquí (DZUP), Porto Alegre (Ruszczyk 1986), Pelotas (Biezanko 1959), Santa Maria (Link et al. 1977), Tapes (DZUP), Vacaria (DZUP), Viamão (Tyler et al. 1994); Santa Catarina: Curitibanos (DZUP). ARGENTINA: Misiones (Canals 2003). URUGUAI: Soriano: Mercedes (DZUP).

Distribuição conhecida no Paraná: Candói, Foz do Jordão, Guarapuava e Pinhão.

Registro inédito no Paraná: Guarapuava, Fazenda Três Capões. $25^{\circ} 24^{\prime}$ S e 51 ${ }^{\circ} 41^{\prime}$ 'W. 938 m (23.I.2007; 25.XI.2007; 03.II.2008).

Biologia: Recentemente, uma população foi encontrada em uma pequena mancha de Campo Natural da Fazenda Três Capões, distante $20 \mathrm{~km}$ a oeste da cidade de Guarapuava. Mais de 40 indivíduos foram avistados em um único dia no mês de fevereiro, indicando uma abundância elevada, embora restrita aos Campos Naturais. Voa nas horas mais quentes a procura de alimento, de parceiros para a cópula e no caso das fêmeas, de sua planta hospedeira, Aristolochia sp. A espécie distribui-se pelos três estados sulinos, podendo ser comum em certas localidades do Rio Grande do Sul (Santa Maria (Link et al. 1977), Vacaria (DZUP)) e Santa Catarina (Curitibanos) (DZUP). No Paraná, limite norte de sua distribuição, sua ocorrência é restrita aos Campos Naturais do terceiro planalto, onde havia sido registrada pela última vez em 21 de novembro de 1986 no município de Foz do Jordão (Mielke \& Casagrande, 2004).

Sugestão de alteração no critério de ameaça: Por sua extensão de ocorrência conhecida ser estimada em menos de $10 \mathrm{~km}^{2}$, deve ser elevada à categoria de criticamente em perigo (CR). Adicionalmente foram observados registros de declínio no número de sub-populações, indicando situação B2b(iv).

Cyanophrys bertha (Jones, 1912) (Lycaenidae: Theclinae) (Figuras 1213).

\section{Situação atual: EN B2ab(ii,iii,iv)}

Distribuição geral: BRASIL: Rio Grande do Sul: Derrubadas (Parque Estadual do Turvo) (Kaminski et al. 2010), Nova Petrópolis (Kaminski et al. 2010) e Santa Rosa (Campus UNIJUI) (Kaminski et al. 2010); Santa Catarina: Corupá (Robbins \& Duarte, 2005); São Paulo: Jundiaí (Serra do Japi) (Brown 1992); Minas Gerais: Barbacena (Robbins \& Duarte 2005) e Poços de Caldas (Ebert, 1969); ARGENTINA: Misiones: Almirante Brown (Reserva Particular Yacutinga) (Núñez Bustos, 2008).

Distribuição conhecida no Paraná: Curitiba e Ponta Grossa.

Registros inéditos no Paraná: Guarapuava, Fazenda Atalaia ( $8 \mathrm{~km}$ ao norte da cidade de Guarapuava), $25^{\circ} 18^{\prime} \mathrm{S}$ e $51^{\circ} 30^{\prime} \mathrm{S}$. 1.075 m (5.I.2008), Guarapuava, Centro (Rua Saldanha Marinho), $25^{\circ} 23^{\prime} \mathrm{S}$ e $51^{\circ} 27^{\prime}$ W. $1.036 \mathrm{~m}$ (XI.1951 e II.1994), Castro (sem localidade exata) (D’A Abrera 1995), 24 47' S e 5000' W. 984 m. e Piên (Trigolândia) (Kaminski et al. 2010).

Biologia: Uma fêmea da espécie foi registrada por volta das 16:00 horas enquanto pousava num arbusto, após descer do dossel da floresta de um pequeno fragmento de Floresta Ombrófila Mista localizado na Fazenda Atalaia, às margens da estrada PR 460. Registros mais antigos para a espécie foram feitos por Hipólito
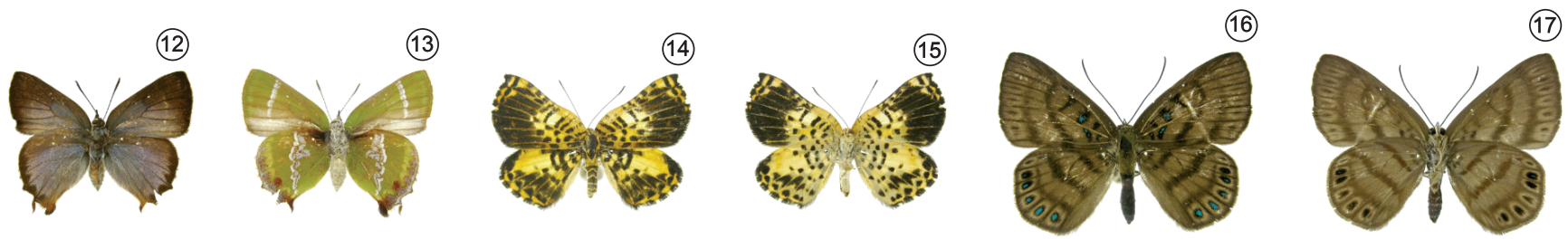

(18)
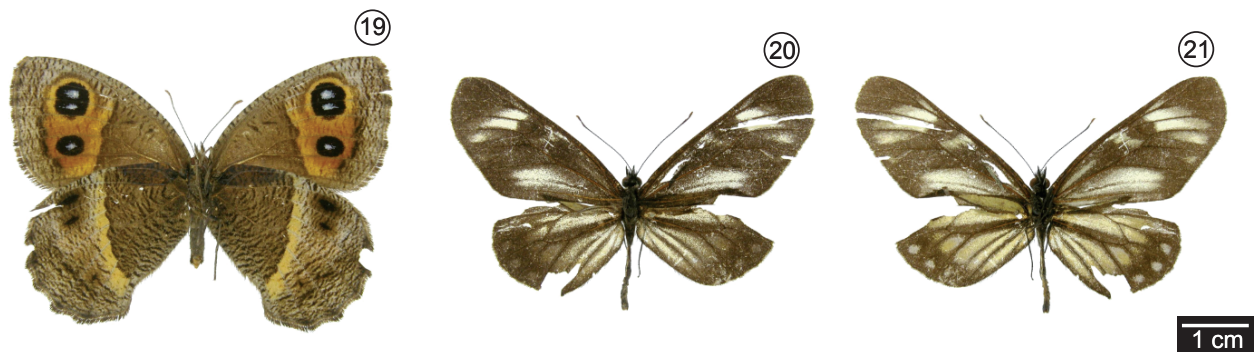

Figuras 12-21. (12-13) Cyanophrys bertha, fêmea (dorsal e ventral) (Guarapuava, centro da cidade); 14-15) Symmachia arion, fêmea (dorsal e ventral) (Parque Estadual do Cerrado, Jaguariaíva); 16-17) Alesa prema, fêmea (dorsal e ventral) (Parque Municipal dos Três Morrinhos, Terra Rica); 18-19) Pampasatyrus glaucope glaucope, fêmea (dorsal e ventral) (Colônia Witmarsum, Palmeira); 20-21) Charonias theano, macho (dorsal e ventral) (Guarapuava).

Figures 12-21. (12-13) Cyanophrys bertha, female (dorsal and ventral) (Guarapuava, downtown); 14-15) Symmachia arion, female (dorsal and ventral) (Parque Estadual do Cerrado, Jaguariaíva); 16-17) Alesa prema, female (dorsal and ventral) (Parque Municipal dos Três Morrinhos, Terra Rica); 18-19) Pampasatyrus glaucope glaucope, female (dorsal and ventral) (Colônia Witmarsum, Palmeira); 20-21) Charonias theano, male (dorsal and ventral) (Guarapuava). 
Schneider em 1951 e 1994, ambos em ambientes já dominados pela expansão urbana. Sua planta hospedeira, Pyrostegia venusta (Ker-Gawl.) Miers (Bignoniaceae) (Kaminski et al. 2010) é comum no fragmento da fazenda, sugerindo a existência de uma população bem estabelecida. Esta espécie é conhecida no estado do Paraná de apenas quatro registros, restritos ao primeiro e segundo planaltos: Castro em meados de 1900 (holótipo depositado no BMNH) (D'Abrera 1995), Curitiba (dezembro de 1945), Ponta Grossa (março de 1948) (Mielke \& Casagrande 2004) e recentemente em Piên (março de 2007) (Kaminski et al. 2010). Os atuais registros ampliam a distribuição da espécie ao terceiro planalto paranaense.

Sugestão de alteração no critério de ameaça: Devido ao aumento no número de localidades e consequente área de ocupação estimada (superior a $2.000 \mathrm{~km}^{2}$ ), a espécie deve ser retirada da lista. Também o declínio continuado aferido na lista vermelha é descaracterizado em todas as três situações B2b(ii,iii,iv).

Symmachia arion (C. Felder \& R. Felder, 1865) (Riodinidae: Riodininae) (Figuras 14-15).

Situação atual: VU B2ab(ii,iii)

Distribuição conhecida: BRASIL: Santa Catarina: São Bento do Sul (DZUP); São Paulo: Jundiaí (Serra do Japi) (Brown 1992); Rio de Janeiro: Petrópolis (DZUP); Minas Gerais: Catas Altas (DZUP) e Poços de Caldas (Ebert, 1969).

Distribuição conhecida no Paraná: Balsa Nova, Colombo, Curitiba, Ponta Grossa e São José dos Pinhais.

Registro inédito no Paraná: Jaguariaíva, Parque Estadual do Cerrado. 24 09' S e 49³9' W. 830 m (19.XI.2009).

Biologia: Durante uma expedição em novembro de 2009, foram observadas duas fêmeas da espécie pousando em galhos secos e folhas em um caminho próximo ao centro de visitação do parque, sempre em horário de sol forte. Este é o primeiro registro da espécie para uma unidade de conservação no estado, e o primeiro para o bioma Cerrado no Brasil. O Parque Estadual do Cerrado possui cerca de 1.800 ha e em sua maior parte é constituída por Cerrado com floresta de galeria a margem dos rios que cruzam a reserva. Registrada pela última vez no município de Balsa Nova, em abril de 1981, esta espécie está sempre atrelada à borda da Floresta Ombrófila Mista com Campo Natural (Mielke \& Casagrande 2004).

Sugestão de alteração no critério de ameaça: Assim como para C. bertha, está espécie deve ser retirada da lista, principalmente pelo aumento no número de localidades e consequente área de ocupação estimada.

Alesa prema (Godart, [1824]) (Riodinidae: Riodininae) (Figuras 1617).

Situação atual: VU B2ab(ii,iii)

Distribuição geral: BRASIL: São Paulo: Teodoro Sampaio (Morro do Diabo) (Mielke \& Casagrande 1998); Rio de Janeiro: Petrópolis (DZUP) e Guapí (DZUP); Espírito Santo: Conceição da Barra (DZUP) e Linhares (DZUP); Minas Gerais: Paracatú (DZUP); Bahia: Rio de Contas (DZUP); Alagoas: Maceió (DZUP); Distrito Federal: Brasília (rio Maranhão) (Brown \& Mielke 1967); Mato Grosso: Barra do Garças (DZUP); Acre: Bujari (DZUP); Amazonas: São Paulo de Olivença (DZUP). ARGENTINA: Misiones: Almirante Brown (Reserva Particular Yacutinga) (Núñez Bustos, 2008) e Puerto Iguazú (Parque Nacional del Iguazú) (Núñez Bustos, 2009).

Distribuição conhecida no Paraná: Foz do Iguaçu e Guaíra.

Registro inédito no Paraná: Terra Rica, Parque Municipal Três Morrinhos. 22 $2^{\circ} 46^{\prime} \mathrm{S}$ e $52^{\circ} 39^{\prime}$ W. 400-615 m (17.XII.2009).
Biologia: Uma única fêmea foi coletada no topo do morro do Parque Municipal Três Morrinhos, por volta das 10:00 horas exibindo comportamento de hilltopping (ver Z. zonia diabo). O mesmo comportamento foi visto nos registros feitos em Teodoro Sampaio (SP) em 1990 e 1991 (Mielke \& Casagrande 1998). Até então a espécie fora registrada no Paraná somente em duas localidades, dentre as quais uma foi extinta (Parque Nacional de Sete Quedas, Guaíra). O presente dado adiciona-se a Foz da Iguaçú (janeiro de 1980) e caracteriza a Floresta Estacional Semidecidual como habitat típico da espécie no estado. Sua planta hospedeira ainda permanece desconhecida.

Sugestão de alteração no critério de ameaça: Sem alteração.

Pampasatyrus glaucope glaucope (C. Felder \& R. Felder, 1865) (Nymphalidae: Satyrinae) (Figuras 18-19).

Situação atual: VU B2ab(ii,iii,iv)

Distribuição geral: BRASIL: Santa Catarina: Urubici (DZUP).

Distribuição conhecida no Paraná: Balsa Nova, Carambeí, Castro, Curitiba, Guarapuava, Palmeira, Ponta Grossa e Tibagi.

Registro inédito no Paraná: Palmeira, Colônia Witmarsun, 252 25' S e 49 49' S. 1.036 m (01.III.1987).

Biologia: Segundo Mielke \& Casagrande (2004), o último registro ocorreu em 1972, no município de Balsa Nova, no entanto uma série de 15 exemplares amostrados em março de 1987, na Colônia Witmarsum, encontra-se na coleção de C. Mielke (CGCM). Essa série representa o registro mais recente para a espécie no Paraná. A espécie não foi mais coletada, mesmo após muitas expedições às regiões de seu registro pretérito, pois as áreas naturais foram eliminadas. Sua ocorrência está atrelada as áreas de Campos Naturais secos.

Sugestão de alteração no critério de ameaça: Mesmo com um novo dado de coleta, este corresponde a um registro de 23 anos. Os critérios B2ab(ii,iii,iv) não se alteraram.

Charonias theano (Boisduval, 1836) (Pieridae: Pierinae) (Figuras 2021).

Situação atual: EN (Paraná) B2ab(ii,iii); EN (lista nacional) B1ab(i,ii,iii,iv)

Distribuição geral: BRASIL: Santa Catarina: Joinville (Casagrande \& Mielke 2008); São Paulo: Avaré (DZUP) e São Paulo (DZUP); Minas Gerais: Cambuquira (DZUP), Conceição dos Ouros (DZUP), Passa Quatro (DZUP), Poços de Caldas (Ebert, 1969) e Pouso Alegre (DZUP).

Distribuição conhecida no Paraná: Cândido de Abreu e Ponta Grossa.

Registro inédito no Paraná: Guarapuava (sem localidade exata), $25^{\circ} 23^{\prime} \mathrm{S}$ e $51^{\circ} 27^{\prime}$ W. $1.030 \mathrm{~m}$ (22.IV.1951).

Biologia: Registro feito com base em um exemplar macho da coleção particular de Hipólito Schneider. Sua inclusão neste estudo se justifica pela adição de mais uma localidade de sua distribuição, até então desconhecida. Desde esse registro, a espécie nunca mais foi encontrada nessa mesma localidade. Provavelmente o exemplar foi amostrado no ecótone entre a Floresta Estacional Semidecidual e a Floresta Ombrófila Mista. Outros dois registros já publicados, de Cândido de Abreu e Ponta Grossa, representam as únicas informações sobre a distribuição da espécie no estado, a qual não é registrada há 51 anos.

Sugestão de alteração no critério de ameaça: Sem alteração. 


\section{Discussão}

A situação atual do conhecimento acerca das espécies ameaçadas de extinção no Estado do Paraná ainda permanece insatisfatória. Dentre as 15 espécies de Lepidoptera citadas no livro vermelho de 2004, apenas 23 localidades são registros de distribuição. De fato, ainda sobram muitas regiões inexploradas cientificamente, e o acentuado grau de desmatamento nessas regiões reduz as possibilidades de estudá-las. Os novos registros apresentados acrescentam seis localidades com ocorrência de alguma espécie de Lepidoptera ameaçada, das quais quatro são do Noroeste do Paraná (Planaltina do Paraná, Terra Rica, Loanda e Paranavaí), uma do nortecentral (Tamarana) e uma do nordeste (Jaguariaíva).

Exceto a região de Guarapuava, todas as espécies registradas localizam-se em unidades de conservação, como Parques Estadual e Municipal, ou Reservas Particulares do Patrimônio Natural. Embora esse aspecto demonstre uma aparente segurança da preservação dessas espécies, é importante mencionar que a localidade em que se inserem é representada por fragmentos altamente reduzidos em tamanho (Passos 2006), não livres de diversos impactos antrópicos, como a caça, extração ilegal de madeira, exploração turística, ou mesmo redução de área total de floresta. Os remanescentes naturais são circundados por extensas áreas de criação de gado e produção de cana-de-açúcar, conferindo um grande risco devido às queimadas e uma acentuada barreira de dispersão para muitas espécies (Passos 2006).

No entanto, Passova passova practa parece não ser afetada negativamente pelos efeitos da fragmentação, cuja grande abundância é frequente em três pequenos fragmentos localizados no noroeste paranaense, alguns deles sob forte influência de efeitos de borda. Curiosamente no Parque Estadual do Morro do Diabo, Mielke \& Casagrande (1998) não observaram a espécie, apesar de se tratar do maior remanescente de Floresta Estacional Semidecídual da região (36.000 ha). O comportamento de vôo dos adultos provavelmente é o fator determinante para ausência de registros da espécie, pois voam sobre o dossel descendo esporadicamente, ou pousando debaixo de folhas no interior da mata. Certamente a espécie existe no Morro do Diabo, mas o fato de ocorrer em pequenos fragmentos reforça a relevância dessas áreas, ainda que pequenas, para a preservação de espécies raras e/ou ameaçadas.

A topografia também é um aspecto que influencia diretamente o comportamento de dispersão e reprodução de borboletas (Pe'er et al. 2004). Sob essa perspectiva, é impossível não mencionar a coocorrência das mesmas espécies ameaçadas nas únicas localidades da formação Arenito Caiuá que contém pequenos morros: o Parque Estadual do Morro do Diabo (Mielke \& Casagrande 1998) e Parque Municipal Três Morrinhos. Em ambos foram registrados Zonia zonia diabo e Alesa prema, devido à manifestação dos seus comportamentos de hilltopping. Neste caso em particular, a manutenção de habitat natural nos topos de morros é fundamental para a sobrevivência dessas e de outras inúmeras espécies de mesmo comportamento, visto que machos podem não reconhecer habitats alterados como áreas convenientes para reprodução (Baughman \& Murphy 1988, Prieto \& Dahners 2006). Além do reduzido tamanho (apenas 11 ha), o Parque Municipal Três Morrinhos tem o seu topo repleto de antenas de comunicação, que reduzem ainda mais a vegetação típica, e rotineiramente recebe acentuada visita de turistas para atividades recreativas e/ou profissionais de vôo de Asa Delta.

Já na região de Guarapuava, a ausência de unidades de conservação, que compreenda os Campos Naturais, talvez seja o fator determinante para a atual situação das espécies características deste ecossistema. A principal justificativa de ameaça dos lepidópteros é recorrente nas listas brasileiras, estaduais e internacionais: a perda de hábitat natural (Casagrande \& Mielke 1995, Brown Jr. 1996, Casagrande et al. 1998, Schmidt et al. 2004, van Swaay et al. 2010). Na região, os Campos Naturais foram quase totalmente convertidos em práticas agropastoris, sendo através de queimadas ou substituição por espécies exóticas de gramíneas. Um exemplo recente retrata a conversão do último remanescente de Campo Natural, contendo os mais recentes registros de Euryades corethrus (2007-2008), em pastagem para gado, provavelmente extinguindo a população local. Certamente é necessária a localização de remanescentes de Campo Natural na região com o intuito de estabelecer unidades de conservação que abrigue tal vegetação, visto a fragilidade de sua fauna e flora típicos.

Também devemos citar a importância do Parque Estadual do Cerrado, que apesar de ser o único grande remanescente dessa vegetação no Paraná, apresenta-se em bom estado de conservação. Como pequenas manchas de campos naturais e matas com araucária são presentes no parque, há influência destes ecossistemas nas áreas de cerrado, a ponto de serem inseparáveis em escalas ecológicas, fazendo com que espécies típicas desses estejam distribuídas pelo parque (Straube et al. 2005). Assim como para algumas espécies de aves (Straube et al. 2005) a presença de Symmachia arion deve decorrer também em função dessas influências, já que a Floresta Ombrófila Mista constitui o habitat da maior parte dos registros para esta espécie.

A maioria das localidades dos registros foi amostrada mais de uma vez, com exceção do Parque Estadual do Cerrado em Jaguariaíva (S. arion) e a Fazenda Atalaia em Guarapuava (C. berha). No entanto, exceto $A$. prema, todos os registros foram feitos na primeira visita de coleta nas áreas. Isso pode indicar que as espécies são comuns em tais localidades, ou ainda, que haja deficiência amostral no estado, especialmente nas regiões mais distantes do único centro de pesquisa que possui pesquisadores de borboletas no Paraná (UFPR - Curitiba), indicando mais uma vez a forte correlação de conhecimento sobre borboletas aos grandes centros urbanos (Carneiro et al. 2008).

Salvo alguns casos (p. ex. Brown Jr. 1972, Otero \& Brown Jr. 1985, Francini et al. 2005, Mielke \& Casagrande 2007), a produção de conhecimento científico acerca dos lepidópteros ameaçados de extinção no Paraná e no Brasil pode ser considerada nula. Se em parte, seu desconhecimento é justificado pela evidente escassez de dados sobre as espécies, por outro lado, a legislação vigente desestimula ainda mais o desenvolvimento de pesquisas envolvendo coletas de animais ameaçados, exigindo do cientista uma demanda burocrática ainda maior que a de rotina para pesquisa. Enquanto essas demandas permanecerem dificultando o acesso à pesquisa, pouco de informação relevante deve ser adicionada às espécies de borboletas ameaçadas no Brasil.

\section{Conclusões}

O pequeno conhecimento atual acerca das espécies de Lepidóptera ameaçadas de extinção no Paraná e Brasil é tão escassa a ponto de pequenas novas informações alterarem substancialmente a situação de ameaça dessas espécies. No entanto, para que novos estudos sejam produzidos no Brasil, é necessário um maior incentivo à pesquisa básica e facilitação da legislação à condução de pesquisas com espécies ameaçadas.

\section{Agradecimentos}

Somos gratos a RPPN Paraná, na pessoa de Anderson Luis Tosetto pelo suporte logístico durante os trabalhos no noroeste do Paraná. Ao Sr. Clóvis Amaral e Sr ${ }^{\mathrm{a}}$. Renata Scommegna, proprietários da RPPN Matão e RPPN Duas Barras, respectivamente, pela disponibilidade de suas áreas a pesquisa. Ao chefe do Parque Estadual do Cerrado, Tadeu Capriotti pela disponibilidade e apoio logístico. Agradecemos também a Luis Anderson Leite e Juliano Cordeiro pelo auxílio em campo. A Fábio Dangui por disponibilizar a coleção particular de Hipólito Schneider. Ao CNPq pela concessão das bolsas de produtividade, de doutorado e de mestrado aos autores. 


\section{Referências Bibliográficas}

BAUGHMAN, J.F. \& MURPHY, D.D. 1988. What constitutes a hill to a hilltopping butterfly? Am. Midl. Nat. 120:441-443.

BERNARDES, A.T., MACHADO, A.B. \& RYLANDS, A.B. 1990 Fauna brasileira ameaçada de extinção. Fundação Biodiversitas, Belo Horizonte.

BIEZANKO, C.M. 1959. Papilionidae da zona sueste do Rio Grande do Sul. Arq. Entomol., Ser. A Ia:1-16.

BROWN Jr., K.S. \& MIELKE, O.H.H. 1967. Lepidoptera of the Central Brazil plateau. I. Preliminary list of Rhopalocera (Continued): Lycaenidae, Pieridae, Papilionidae e Hesperiidae. J. Lepid. Soc. 21(3):145-168.

BROWN JR., K.S. 1972. The Heliconians of Brazil (Lepidoptera: Nymphalidae). Part III. Ecology and biology of Heliconius nattereri, a key primitive species near extinction, and comments on the evolutionary development of Heliconius and Eueides. Zoologica-NY 57:41-69.

BROWN Jr., K.S. 1992. Borboletas da Serra do Japi: Diversidade, habitats, recursos alimentares e variação temporal. In História natural da Serra do Japi: Ecologia e preservação de uma área florestal no sudeste do Brasil (L.P.C. Morellato, ed). Unicamp; FAPESP, Campinas, p. 142-187.

BROWN Jr., K.S. 1996. Conservation of threatened species of Brazilian butterflies. In Decline and Conservation of Butterflies in Japan (S.A.A.T. Hirowatari, M. Ishii \& L.P. Brower, eds.). Lepidopterists Society of Japan, Osaka, v. 3, p. 45-62.

CANALS, G. 2003. Mariposas de Misiones. L.O.L.A., Buenos Aires, 476p.

CARNEIRO, E., MIELKE, O.H.H. \& CASAGRANDE, M.M. 2008. Inventário de borboletas no Brasil: estado da arte e modelo de áreas prioritárias para pesquisa com vistas à conservação. Nat. Conserv. 6(2):6890. http://internet.boticario.com.br/Internet/staticFiles/Fundacao/pdf/ ARTIGOS_NC12/11N\&C_V6_N2_SANTOS_PORT.pdf

CASAGRANDE, M.M. \& MIELKE, O.H.H. 1993. Borboletas (Lepidoptera) ameaçadas de extinção no Paraná. Rev. Bras. Zool. 9:75-92.

CASAGRANDE, M.M. \& MIELKE, O.H.H. 1995. Borboletas Ameaçadas de Extinção no Estado Paraná. In Lista Vermelha de Animais Ameaçados de extinção no Estado do Paraná (SEMA, org.). SEMA/GTZ, Curitiba, p. $143-157$

CASAGRANDE, M.M. \& MIELKE, O.H.H. 2008. Lepidoptera: Capítulo 46 - Charonias theano theano (Boisduval, 1836). In Livro Vermelho da fauna ameaçada de extinção. (A.B.M. Machado, G.M. Drummond \& A.P. Plagia, org.). Biodiversidade 19(1):438-439.

CASAGRANDE, M.M., MIELKE, O.H.H. \& BROWN JR., K.S. 1998. Borboletas (Lepidoptera) ameaçadas de extinção em Minas Gerais, Brasil. Rev. Bras. Zool. 15:241-259.

D'ABRERA, B. 1995. Butterflies of the Neotropical Region. Part VII. Lycaenidae. Hill House, Victoria, p. 1098-1270.

EBERT, H. 1969. On the frequency of butterflies in eastern Brazil, with a list of butterfly fauna of Poços de Caldas, Minas Gerais. J. Lepid. Soc. 23(Suppl 3):1-48.

FONTANA, C.S., BENCKE, G.A. \& REIS, R.E. 2003. Livro vermelho da fauna ameaçada de extinção no Rio Grande do Sul. EDIPUCRS, Porto Alegre, p.632.

FRANCINI, R.B., FREITAS, A.V.L. \& BROWN Jr., K.S. 2005. Rediscovery of Actinote zikani (D'Almeida) (Nymphalidae, Heliconiinae, Acraeini): natural history, population biology and conservation of an endangered butterfly in SE Brazil. J. Lepid. Soc. 59(3):134-142.

IUCN. 2001. IUCN Red List Categories: version 3.1. Prepared by the IUCN Species Survival Commission. IUCN, Gland, Switzerland and Cambridge, UK.

KAMINSKI, L.A., THIELE, S.C., ISERHARD, C.A., ROMANOWSKI, H.P. \& MOSER, A. 2010. Natural history, new records, and notes on the conservation status of Cyanophrys bertha (Jones) (Lepidoptera: Lycaenidae). P. Entomol. Soc. Wash. 112:54-60.

LAMAS, G. 2004. Checklist: Part 4A Hesperioidea - Papilionoidea. In Checklist of Neotropical Lepidoptera. (J. Heppner, org.). Association of Neotropical Lepidoptera, Gainesville, p. 439
LINK, D., BIEZANKO, C.M., TARRAGÓ, M.F. \& CARVALHO, S. 1977. Lepidoptera de Santa Maria e arredores. I Papilionidae e Pieridae. Rev. Cent. Cienc. Rur. 7:381-389.

MACHADO, A.B.M., FONSECA, G.A.B., MACHADO, R.B., AGUIAR, L.M.S. \& LINS L.V. (eds.). 1998. Livro Vermelho das Espécies Ameaçadas de Extinção da Fauna de Minas Gerais. Fundação Biodiversitas, Belo Horizonte, 605p.

MIELKE, O.H.H. \& CASAGRANDE, M.M. 1998. Papilionoidea e Hesperioidea (Lepidoptera) do Parque Estadual do Morro do Diabo, Teodoro Sampaio, São Paulo, Brasil. Rev. Bras. Zool. 14:966-1001.

MIELKE, O.H.H. \& CASAGRANDE, M.M. 2004. Borboletas In Livro Vermelho da Fauna Ameaçada no Estado do Paraná (S.B. Mikich \& R.S. Bernils, org). Instituto Ambiental do Paraná, Curitiba, p. 713-739.

MIELKE, O.H.H. \& CASAGRANDE, M.M. 2007. Parides klagesi (Ehrmann) redescoberto no Brasil (Lepidoptera, Papilionidae, Troidini). Rev. Bras. Ent. 51:187-189.

MIKICH, S.B. \& BERNILS, R.S. (ed.). 2004. Livro Vermelho da Fauna Ameaçada no Estado do Paraná. Instituto Ambiental do Paraná, Curitiba.

NÚÑEZ BUSTOS, E.O. 2008. Diversidad de mariposas diurnas en la Reserva Privada Yacutinga, Provincia de Misiones, Argentina. Trop. Lep. Res. 18(2):78-87.

NÚÑEZ BUSTOS, E.O. 2009. Mariposas diurnas (Lepidoptera: Papilionoidea y Hesperioidea) del Parque Nacional Iguazú, Provincia de Misiones, Argentina. Trop. Lep. Res. 19:71-81.

OTERO, L.S. \& BROWN Jr., K.S. 1985. Biology and ecology of Parides ascanius (Cramer, 1775) (Lep., Papilionidae), a primitive butterfly threatened with extinction. Atala 10/12:2-16.

PARANÁ. SEMA. (Org.). 1995. Lista Vermelha de Animais Ameaçados de extinção no Estado do Paraná. SEMA/GTZ, Curitiba.

PASSOS, M.M. 2006. Das potencialidades à evolução paisagística no noroeste do Paraná: uma aproximação. Geografia 15:173-204.

PE'ER, G., SALTZ, D., THULKE, H.H. \& MOTRO, U. 2004. Response to topography in a hilltopping butterfly and implications for modeling nonrandom dispersal. Anim. Behav. 68:825-839.

PRIETO, C. \& DAHNERS, H.W. 2006. Eumaeini (Lepidoptera: Lycaenidae) del cerro San Antonio: Dinámica de la riqueza y comportamiento de "Hilltopping". Rev. Colomb. Entomol. 32:179-190.

ROBBINS, R.K. \& DUARTE, M. 2005. Phylogenetic analysis of Cyanophrys Clench, a synopsis of its species, and the potentially threatened $\mathrm{C}$. bertha (Jones) (Lycaenidae: Theclinae: Eumaeini). P. Entomol. Soc. Wash. 107:398-416.

RUSZCZYK, A. 1986. Distribution and abundance of butterflies in the urbanization zones of Porto Alegre, Brazil. J. Lepid. Soc. 25(3):157-178.

SCHMIDT, P., SCHÖNBORN, C., HÄNDEL, J., KARISCH, T., KELLNER, J. \& STADIE, D. 2004. Rote Liste der Schmetterlinge (Lepidoptera) des Landes Sachsen-Anhalt. Berichte des Landesamtes für Umweltschutz Sachsen-Anhalt 39:388-402.

SHIELD, O. 1967. Hilltopping. An ecological study of summit congregation behavior of butterflies on a southern California hill. J. Res. Lepidoptera 6:69-178.

STRAUBE, F.C., URBEN-FILHO, A. \& GATTO, C. 2005. A avifauna do Parque Estadual do Cerrado (Jaguariaíva, Paraná) e a conservação do cerrado em seu limite meridional de ocorrência. Atual. Ornitol. 127:29-49.

TYLER, H., BROWN Jr., K.S. \& WILSON, K. 1994. Swallowtail Butterflies of the Americas: a Study in Biological Dynamics, Ecological Diversity, Biosystematics, and Conservation. Gainesville, Scientific Publishers, $377 \mathrm{p}$.

Van SWAAY, C., CUTTELOD, A., COLLINS, S., MAES, D., MUNGUIRA, M.L., ŜASIĆ, M., SETELLE, J., VEROVNIK, R., VERSTRAEL, T., WARREN, M., WIEMERS, M. \& WYNHOFF, I. 2010. European Red List of Butterfly. Publications Office of European Union, Luxembourg.

Recebido em 13/04/2010 Versão reformulada recebida em 16/06/2010 Publicado em 08/07/2010 